\title{
Extremely Thin Metal Foil Blades as Cutting Tools for Hard and Brittle Materials
}

\author{
Satoshi Sakamoto ${ }^{1}$, Sanshiro Akaoka ${ }^{1}$, Masaya Gemma ${ }^{1}$, Yasuo Kondo ${ }^{2}$, Kenji Yamaguchi ${ }^{3}$, Mitsugu Yamaguchi ${ }^{4}$ and \\ Takao Yakou ${ }^{1}$ \\ ${ }^{1}$ Yokohama National University, 79-2 Tokiwadai, Hodogaya-ku, Yokohama, Japan \\ ${ }^{2}$ Yamagata University, 4-3-16 Jonan, Yonezawa, Japan \\ ${ }^{3}$ Yonago National College of Technology, 4448 Hikona-cho, Yonago, Japan \\ ${ }^{4}$ Salesian Polytechnic, 4-6-8 Oyamagaoka, Machida, Japan
}

\begin{abstract}
The manufacturing costs of semiconductor products such as silicon wafers can be reduced by decreasing the kerf loss. In addition, a decrease in the kerf loss leads to an effective utilization of rare materials, which is environmentally beneficial from the viewpoint of saving resources. This study aims to reduce the kerf loss during slicing hard and brittle materials. Therefore, the possibility of using an extremely thin metal foil blade instead of a wire tool in slicing was examined. Initially, grooving characteristics using a metal foil blade (thickness: $50 \mu \mathrm{m}$ or less) was investigated. The main conclusions are that grooving with a metal foil blade is possible and kerf loss can be reduced. The groove depth tends to increase as the machining time and particle size of abrasives increase. The groove width is smaller when a thin metal foil blade is used and vice versa. However, if the abrasive particle size is too large, grooving becomes impossible. Since the wear of metal foil blade increases with an increase in the particle size of the abrasive, it is necessary to use an abrasive with a particle size that is suitable for the thickness of the metal foil blade.
\end{abstract}

\section{Introduction}

Multi-wire sawing is an excellent slicing method for hard and brittle materials, such as silicon ingots, magnetic materials, ceramics, and sapphire [1]. In recent years, multi-wire sawing has emerged as the mainstream slicing method for ingots with large diameter. In addition, new types of wire tools and slicing devices are being developed [2-4]. Currently, when several thin layers are sliced from an ingot using a multi-wire saw, the volume of kerf loss is same or more than the volume of the wafers [5]. Large kerf loss during slicing not only increases the production cost of semiconductor products and optical parts but also is an important problem from the viewpoint of saving resource [6]. The diameters of the wire tool and particle size of the abrasives that are used for slicing greatly affect the volume of kerf loss. In other words, when the wire tool is thin and particle size is small, the kerf loss can be drastically reduced. However, the wire tool with a small diameter easily breaks during slicing. Therefore, it is very difficult to further reduce the diameter of the wire tool.

This study aims to drastically reduce the kerf loss by examining the possibility of a machining method that utilizes extremely thin metal foil blades instead of a wire tool. In this study, as a first step to realize slicing with reduced kerf loss, we discuss the fundamental grooving characteristics of using an extremely thin metal foil blade and fine abrasives.

\section{Experimental procedure}

Figure 1 shows a schematic illustration of the grooving experimental apparatus, and Figure 2 shows details of the machining section. The materials used and main experimental conditions are summarized in Table 1. The metal foil blade was fixed to the tool holder and pressed against the workpiece under a constant load (machining load). A reciprocating motion was applied to the metal foil blade and grooving experiments were performed. In the grooving experiments, $5 \mathrm{~mm}$ thick sheets of borosilicate glass (TEMPAX) were arranged side by side (Work [A] to [D] in Figure 2). Various measurements were made where work [B] and [C] were machined at a steady state. A 30 $\mathrm{mm}$ long metal foil blade made from alloy tool steel (SKS 81 ) with a thickness between 10 and $50 \mu \mathrm{m}$ was used with a diamond paste of low viscosity, and the particle size of abrasives was between 1 and $6 \mu \mathrm{m}$. The Vickers hardness of the metal foil blade was adjusted to $780 \mathrm{HV}$ by heat treatment. The protrusion of the metal foil blade from the holder was $2.5 \mathrm{~mm}$ for blades with a thickness between 10 and $30 \mu \mathrm{m}$, and $5 \mathrm{~mm}$ for blades with a thickness of $50 \mu \mathrm{m}$. The machining load was $0.1,0.3$, and $0.5 \mathrm{~N}$ for metal foil blades of thickness 10,30 , and $50 \mu \mathrm{m}$, respectively. The relative speed between the metal foil blade and the workpiece was $1212 \mathrm{~mm} / \mathrm{min}$, and the moving distance of the metal foil blade was $100 \mathrm{~mm}$. 


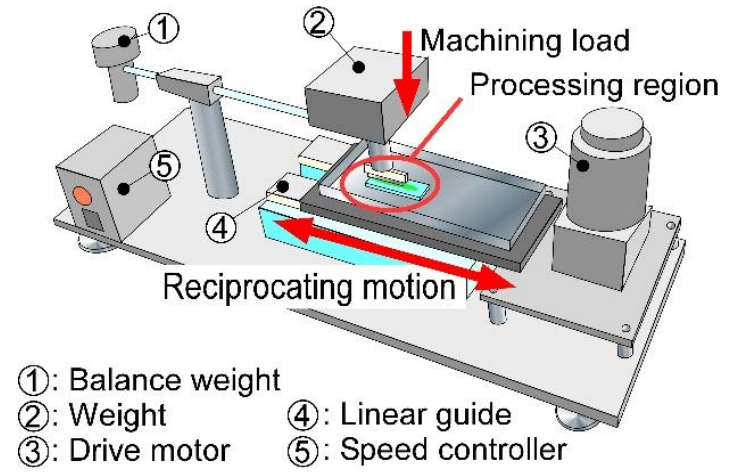

Figure 1. Schematic illustration of grooving experimental apparatus.

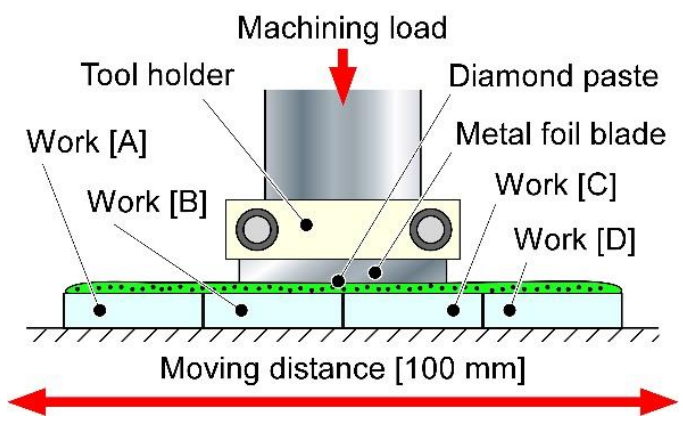

Figure 2. Details of machining section.

Table 1. Materials and experimental conditions of grooving experiments.

\begin{tabular}{ll}
\hline Blade material & Alloy tool steel (SKS81) \\
$\begin{array}{ll}\text { Dimensions } \\
\text { Protrusion }\end{array}$ & Length: $30[\mathrm{~mm}]$, Thickness: 10,30, and $50[\mu \mathrm{m}]$ \\
\hline Abrasive & Diamond \\
Particle size & 1,3 , and $6[\mu \mathrm{m}]$ \\
\hline Work material & Borosilicate glass $(\mathrm{TEMPAX})$ \\
Cross section & Rectangle $(5 \times 20[\mathrm{~mm}])$ \\
\hline Table speed & $1212[\mathrm{~mm} / \mathrm{min}]$ \\
Moving distance & $100[\mathrm{~mm}]$ \\
Machining load & $0.1,0.3$, and $0.5[\mathrm{~N}]$ \\
Machining time & $30,60,90$, and $120[\mathrm{~min}]$ \\
\hline
\end{tabular}

\section{Experimental results and discussion}

\subsection{Example of a groove machined using a metal foil blade}

Figure 3 shows an example of a groove machined using a metal foil blade. The thickness of the metal foil blade was

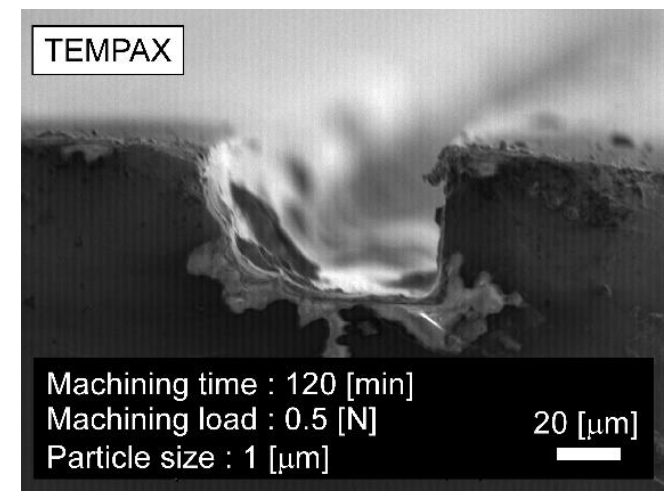

Figure 3. Example of groove machined using a metal foil $(50 \mu \mathrm{m})$.
$50 \mu \mathrm{m}$ and a machining load of $0.5 \mathrm{~N}$ was applied for 120 minutes using an abrasive with a particle size of $1 \mu \mathrm{m}$. One side of the groove was relatively vertical while the other surface was slightly oblique. In this study, the metal foil blade was not tensioned and slight deflection of the metal foil blade due to the machining load was expected. Therefore, one side of the groove is inclined, but it is clear that a hard and brittle material like borosilicate glass can be machined using an extremely thin metal foil blade.

\subsection{Influence on groove depth}

Figure 4 shows the relation between machining time and groove depth. When the thickness of the metal foil was 10, 30 , or $50 \mu \mathrm{m}$, the applied machining load was $0.1,0.3$, or $0.5 \mathrm{~N}$, respectively. In all of these grooving experiments, the particle size of the abrasive was $1 \mu \mathrm{m}$. The groove depth tends to increase with an increase in the machining time. The machining load is several times different depending on the thickness of the metal foil blade. However, there is a slight difference in the depth of the groove, but there is no remarkable difference. This is simply explained because although the applied machining load was different for each blade, the actual machining pressure remained the same in all cases. 
We also investigated the influence of the particle size of the abrasive on the groove depth using the $50 \mu \mathrm{m}$ thick metal foil blade. Figure 5 shows the relation between the particle size and groove depth after a machining time of 120 minutes at an applied load of $0.5 \mathrm{~N}$. The groove depth tends to increase linearly as the particle size increases.

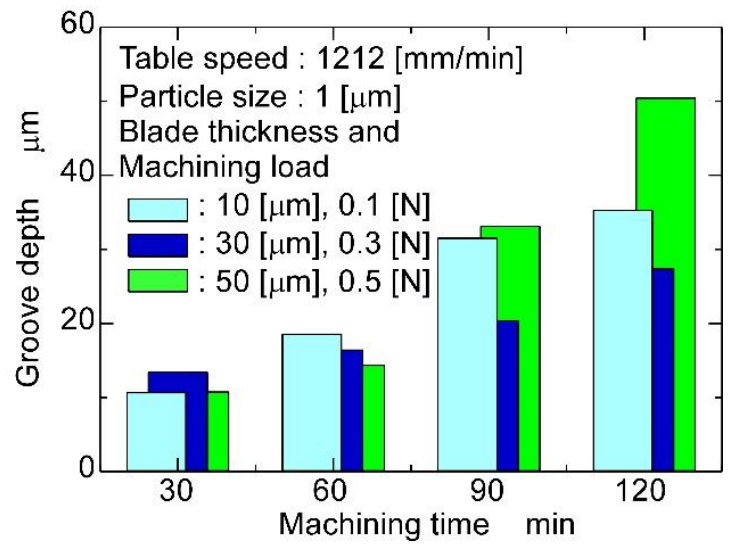

Figure 4. Relationship between machining time and groove depth as observed for three different blade thicknesses.

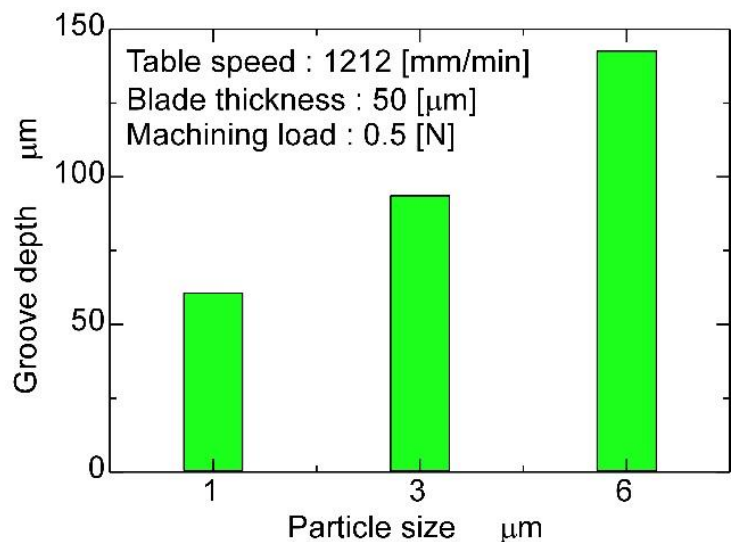

Figure 5. Relationship between the particle size of the abrasive and the groove depth manufactured from a blade with a thickness of $50 \mu \mathrm{m}$.

\subsection{Influence on groove width}

Figure 6 shows the influence of the thickness of the metal foil on the groove width (kerf width). Naturally, the groove width is directly related to the volume of kerf loss. The empirical formula for the width of a groove cut using a multi-wire saw is known [7]. Here, the groove width was calculated using the thickness of the metal foil blade instead of the diameter of the wire tool. Therefore, the calculated groove width is given by equation (1).

$W=T+3 G$,

where $W$ is the calculated groove width, $T$ is thickness of the metal foil, and $G$ is particle size of the abrasive. $W$ is called a calculated value in this study and is indicated by the dashed lines, as shown in Figure 6. It is clear from this figure that the groove width depends on the thickness of metal foil blade. In addition, the groove width is wider than the calculated value. Generally, the groove width in these experiments tends to be larger than the calculated value because it is influenced by the accuracy of the experimental apparatus

A thinner blade is clearly more advantageous for narrowing the groove width. Therefore, the influence of the particle size on the groove width was also investigated using a thin metal foil blade having a thickness of $10 \mu \mathrm{m}$. Figure 7 shows the influence of the particle size on the groove width. The larger the diameter of the abrasive used, the wider the resulting groove width. However, grooving becomes impossible if the particle size is too large such as $6 \mu \mathrm{m}$. It appears that if the particle size of the abrasive is too large relative to the thickness of the metal foil blade, it becomes difficult to efficiently convey the abrasive to the machining section. Therefore, if an abrasive with large particle size is used, grooving becomes impossible. For efficient grooving, it is necessary to use the abrasive with the most suitable particle size in relation to the thickness of the metal foil blade that is being employed.

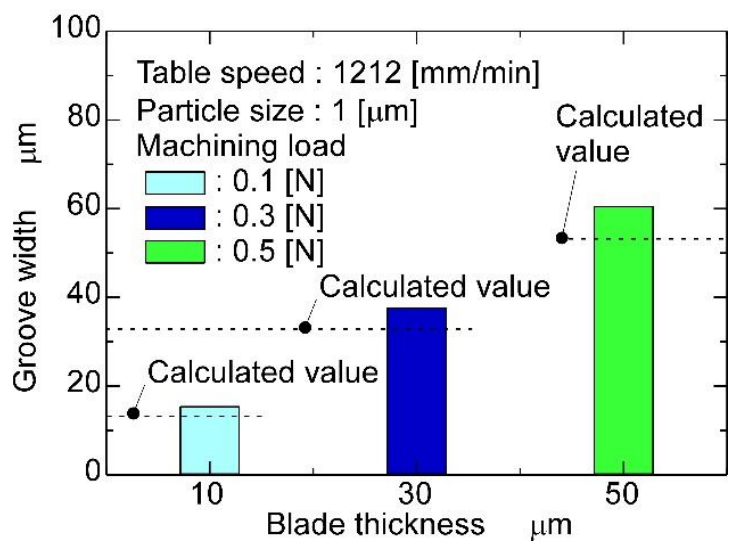

Figure 6. Influence of the thickness of the metal foil on the groove width using a $1 \mu \mathrm{m}$ particle abrasive.

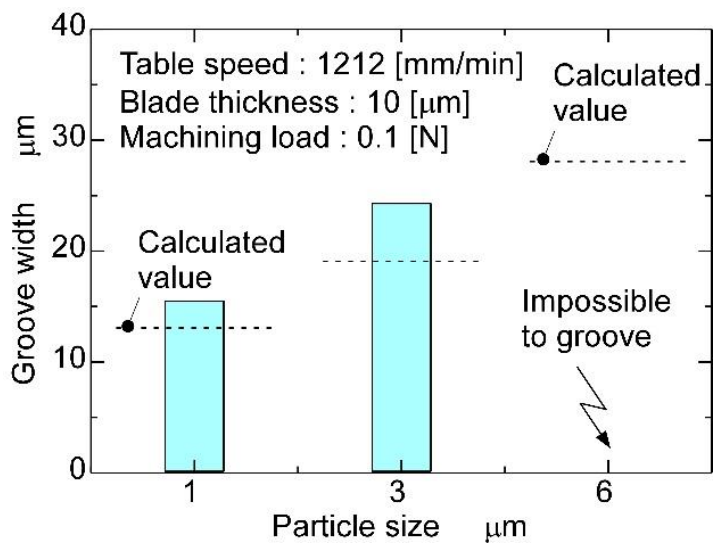

Figure 7. Influence of the particle size on the groove width cut using a $10 \mu \mathrm{m}$ thick metal foil blade.

\subsection{Influence on tool wear}

SEM images and thickness analyses of the $50 \mu \mathrm{m}$ metal foil blade after 60 minutes of machining are shown in Figure 8 and Figure 9, respectively. In Figure 8, the relation between the particle size of the abrasive and effect on the wear of the metal foil blade can be clearly seen. The thickness of the metal foil blade used was $50 \mu \mathrm{m}$. The wear width is the distance from the edge of the blade. The average value was adopted in this study, and it is clear that 
the larger the particle size, larger is the increase in wear width. Furthermore, the wear width of the metal foil blade is about 1.5 to 2 times the particle size of the abrasive used.

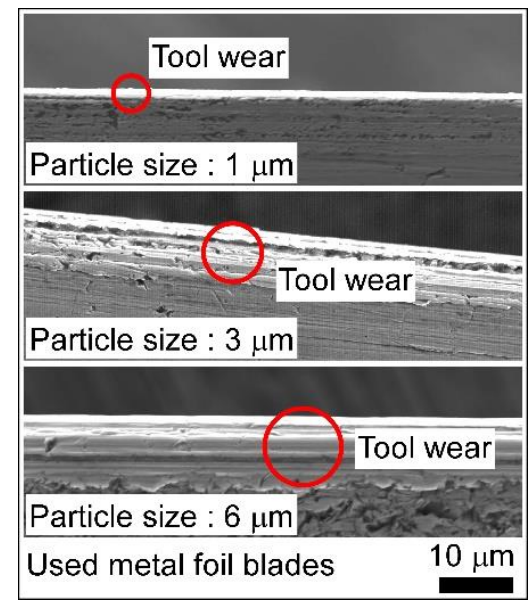

Figure 8. SEM images of a $50 \mu \mathrm{m}$ metal foil blade after machining a groove using 1,3 , or $6 \mu \mathrm{m}$ particle sized abrasives.

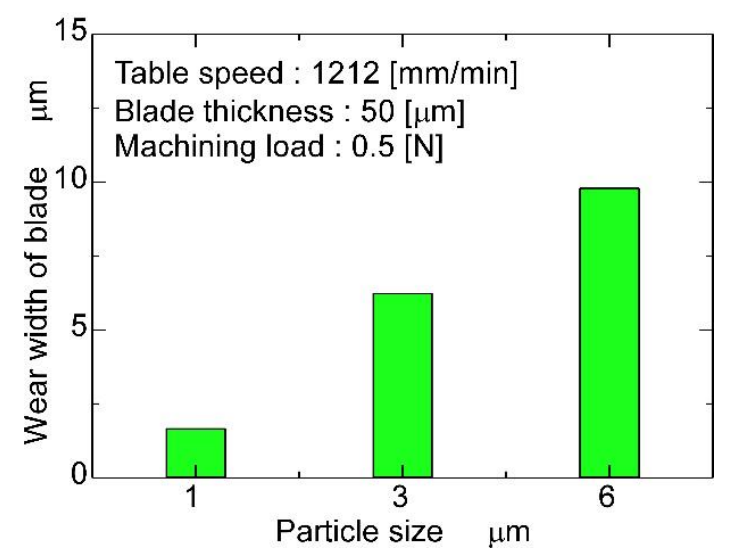

Figure 9. Relationship between the wear width on a $50 \mu \mathrm{m}$ metal foil blade and the particle size of the abrasive used.

\section{Conclusions}

In this study, we experimentally investigated the possibility of using a metal foil blade with a thickness of $50 \mu \mathrm{m}$ or less as a cutting tool for hard and brittle materials.
We demonstrated that the precise machining of borosilicate glass using an extremely thin metal foil blade and fine abrasives is possible. The groove depth tends to increase with both the machining time and particle size of the abrasive. The width of the groove depends on the thickness of the metal foil blade. In other words, the groove width is less when a thinner metal foil blade is used, resulting in a reduced kerf loss. The larger the diameter of the abrasive used, the wider the groove width. However, using an abrasive with too large a particle size will make grooving impossible. Therefore, it is necessary to use an abrasive with a particle size suitable for the thickness of the metal foil blade. In addition, the wear of the metal foil blade increases as the particle size increases. In this study, we could reduce groove width (kerf width) to about $15 \mu \mathrm{m}$ by using an extremely thin metal foil and fine abrasives. Therefore, metal foil processing can be expected to greatly reduce the manufacturing cost of semiconductor wafers and optical parts.

\section{Acknowledgments}

We thank the Instrumental Analysis Center of Yokohama National University for their support and cooperation. This work was supported by JSPS KAKENHI Grant Number $16 \mathrm{~K} 06005$.

\section{References}

[1] H Wu 2016 Precision Engineering 43 1-9

[2] Kamiya $\mathrm{O}$ et al. 2010 International Journal of the Society of Materials Engineering for Resources 17 182-5

[3] Saito A et al. 2014 International Journal of the Society of Materials Engineering for Resources 20 181-5.

[4] Suzuki T et al. 2017 Precision Engineering 50 32-43

[5] Hoshiyama T 2009 J. of the Japan Society for Abrasive Technology 53 655-8

[6] NEDO 2009 Roadmap (PV2030+) (Tokyo: New Energy and Industrial Technology Development Organization)

[7] Kinutani K and Kanamichi 1996 Electronic Materials and Parts 35 (Tokyo: Kogyo Chosakai Publishing Co., Ltd.) 29-33. 